\title{
IOT BASED APPROACH FOR REMOTELY MONITORING AND ALARMING A DROWSY DRIVER
}

\author{
Mr. Aniket Ashok Bhamani ${ }^{1}$, Mr. Sanyam Sanjay Mehta ${ }^{2}$ \\ 1,2Department of Computer Engineering, Vasantadada Patil Pratishthan's College of Engineering \\ \& Visual Arts, Mumbai, Maharashtra, India
}

Article DOI: https://doi.org/10.36713/epra7217 DOI No: 10.36713/epra7217

\begin{abstract}
There are a lot of road accidents that occur due to drowsy driving. Drowsy driving is when the driver of a vehicle is found to be sleepy and probable to get into a car crash because of the same. Being drowsy might cause the driver to lose concentration from the road, and also reduce the reaction time. Statistics suggest how thousands of deaths and crashes happen every year due to it. Major victims of such crashes tend to be the commercial drivers who need to drive long distances overnight. Our project intends to propose a solution to this problem by providing an Internet of Things based approach. This approach monitors the driver's face while he or she is driving the vehicle and in case if the driver is to be found falling asleep, an instant voice call is made to the driver's registered phone number. Additionally, a text message is also sent to the driver's emergency contact which will get him/her notified and provide the driver with quick assistance if needed. This approach is unique and different in its own way as it provides cross platform support and remote monitoring of the driver. Additionally, it also makes drowsy-detection 'device independent'. It offers a simplified mechanism to derive real time accurate results and readings with reduced complexities. This project does have a lot of scope, especially considering that there is a lack of methodologies currently being implemented to prevent road accidents due to drowsy driving.
\end{abstract}

KEYWORDS- Drowsy Driving, Monitoring, Machine Learning, Internet of Things, Remote, Algorithm, Eye Aspect Ratio, Python.

\section{INTRODUCTION}

In recent times, one of the leading causes of road accidents is drowsy driving. NHTSA (National Highway Safety Administration) estimates that it contributes to more than one lakh road crashes every year, having 71,000 people injured and more than 1550 deaths. It also states how about $10-20 \%$ of the road accidents are directly or indirectly a result of drowsy driving. According to the AAA Foundation of Traffic Safety Study, 2 out of every 5 drivers have admitted to falling asleep at some point while driving.

Almost all of the statistics looked at, suggest how drowsy drivers are additionally prone to encountering accidents. Additionally, some studies even suggest that the prevalence of drowsy driving fatalities is $350 \%$ greater than reported. Common victims to this are the commercial drivers who drive trucks, buses, tractors for long distances overnight, and also several night shift workers. Governments in most countries implement fines for violating road safety protocols. Their most policies deal with checking and penalizing people on things such as over speeding, jumping signals, getting caught driving without a driver's license etc. None of these policies seem to be enough to contract accidents caused due to drowsy driving. Additionally, drivers can choose to be careful and take precautions such as- regular coffee breaks, use alerting systems, making sure to get enough sleep before driving, making sure he/she is not under the influence of alcohol while driving etc. However, these safety protocols are not being followed and the drowsy driving victim statistics serve to be evidence for it. Hence, there needs to be a better approach taken towards preventing the accidents caused due to drowsy driving. Table 1. shows the statistics of the Causes of Road Accidents. 
EPRA International Journal of Research and Development (IJRD)

Volume: 6 | Issue: 6 | June 2021

- Peer Reviewed Journal

\begin{tabular}{|l|l|l|}
\hline Behaviour & Number & Percent \\
\hline Driving Too Fast For Conditions or in Excess of Posted Limit or Racing & 8,596 & $16.7 \%$ \\
\hline Under the Influence of Alcohol, Drugs, or Medication & 5,175 & 10.1 \\
\hline Failure to Keep in Proper Lane & 3,706 & 7.2 \\
\hline Failure to Yield Right of Way & 3,579 & 7.0 \\
\hline Distracted (Phone, Talking, Eating, Object, etc.) & 2,688 & 5.2 \\
\hline Operating Vehicle in a Careless Manner & 2,797 & 5.4 \\
\hline Failure to Obey Traffic Signs, Signals, or Officer & 1,990 & 3.9 \\
\hline Operating Vehicle in Erratic, Reckless or Negligent Manner & 1,955 & 3.8 \\
\hline Overcorrecting/Oversteering & 1,617 & 3.1 \\
\hline Vision Obscured Rain, Snow, Glare, Lights, Building, Trees, etc.) & 1,540 & 3.0 \\
\hline Driving Wrong Way on One-Way Trafficway or Wrong Side of Road & 1,243 & 2.4 \\
\hline Drowsy, Asleep, Fatigued, Ill, or Blacked Out & 1,221 & 2.4 \\
\hline Swerving or Avoiding Due to Wind, Slippery Surface, etc. & 1,176 & 2.3 \\
\hline Making Improper Turn & 635 & 1.2 \\
\hline Other Factors & 5,203 & 10.1 \\
\hline None Reported & 9,167 & 17.8 \\
\hline Unknown & 16,012 & 31.1 \\
\hline Total drivers (1) & $\mathbf{5 1 , 4 9 0}$ & $\mathbf{1 0 0 . 0 \%}$ \\
\hline
\end{tabular}

A. Proposed Solution

A real time monitoring and alerting system could possibly offer a solution to this problem. With this project, what we aim at building is a prototype that could monitor the state of the driver and potentially prevent an accident from occurring even before it does. Our project carries out an examination of the driver's face while he/she is driving the vehicle and predicts if the driver is in a sleepy or drowsy state. This is done by running algorithms to compute the aspect ratio of the eyes of the driver. If he/she is found to be drowsy, an instant voice call is placed on the registered mobile number of the user which would alert the driver and make him/her focus on the road again. This project is implemented on the concept of Internet of Things and incorporates several machine learning algorithms in order to render real time accurate results. Additionally, this project takes into consideration the chances of the driver getting into an accident and offers assistance to that as well by notifying the emergency contact of the driver. This can prove to be extremely beneficial for the emergency contact to reach out to the driver on time.
B. Uniqueness of this Approach.

1) Cross Platform

This project is completely based on accessing the camera of the device remotely. It is independent of the underlying platforms, such as Android, IOS etc. All of the functionalities which are provided are not platform specific and can be dispensed on any device irrespective of the operating system being used by that device.

\section{2) Device Independent:}

In addition to being cross platform, this project is designed in such a way that it does not matter what the monitoring device is, it can be a cell phone, a tablet etc. As long as the device supports the minimum requirements of having a camera, calling features and a stable internet connection, this project can be used to successfully alert a sleepy driver and prevent accidents.

\section{3) Simplicity:}

One of the major aspects of this project is that it is kept as simple as possible in terms of its requirements and implementation. All it needs is a device with an integrated camera, active internet 


\section{EPRA International Journal of Research and Development (IJRD)

connection and calling features, which most of the devices these days already possess. Additionally, the algorithms are designed to be implemented using only the libraries and programming concepts that are absolutely required and relevant to alert the driver. Not only does this project successfully avoid the unwanted complexities, but also makes it simple and convenient for the user to understand and use.

\section{4) Remote Monitoring:}

This project aims at providing remote monitoring of the driver. So, there can be someone else sitting at a different location that can monitor the driver while the driver is driving the vehicle. Since most of the drowsy driver accidents happen among youngsters, the remote interface can be used by parents to keep an eye on their kid while he/she is driving. Further, the remote access to the interface can be handed over to someone else before planning a late-night drive as in the case of commercial truck and bus drivers.

\section{5) Victim Support:}

By messaging the emergency contact of the driver, this project makes him/ her check up on the driver by letting him/her know that the driver is not in a stable state to be driving the vehicle. In case if the driver gets into an accident, quick assistance can be provided by the emergency contact and the driver can be taken care of. Hence, this application takes into consideration the aspect of providing assistance to the victim if he/she ends up getting into an accident due to drowsy driving.

\section{LITERATURE SURVEY}

Studies from several accredited sources were conducted and considerations on what the application should be focused on were derived accordingly. Analysis was done on their implementations, advantages, disadvantages and requirements. Conclusions were drawn and efforts were made to incorporate necessary features into our project that the studied implementations lacked and offered limitations to.

\section{A. Government and the Road Transport Authority} approach.

Present system for Road Safety as proposed by the Government and RTA (Road Transport Authority) was studied. A study was done on their policies, the way they were implemented and a detailed research was carried out on their efficiency. The fine structure implemented by the Government on different things to ensure road safety was also studied. Analysis was done on how the system, policies and fines had an impact on the driver's behaviour and whether such a method was enough to reduce the accidents caused due to drowsy driving. Results were drawn to conclude that the present system, present implementations and initiatives made by the RTA and the Government lacked what it takes to prevent a drowsy driver from getting into an accident.

\section{B. Technical Implementations.}

Additionally, other similar technical implementations were looked at. There were quite a few implementations found which worked towards preventing a driver from falling asleep. However none were found, that were IOT based and connected with the driver personally to provide assistance. Also, applications failed to provide victim support as they failed to reach out to the emergency contact of the victim.

1. Program Scripts:

Most of the implementations carried out were merely programmed scripts which can only run on a computer with an appropriate compiler present. Even though this does serve the purpose of monitoring the driver and alarming him/her, it is simply not feasible to be implemented in real life by a driver. An example of this would be the Deep RDD script that can be found online on different platforms including GitHub. Not only was an implementation method of this script missing, but also it was found to have a lot of interdependencies which made it computationally expensive. Overall, it was concluded that such a script could not be workable by a driver. However, using similar scripts like this one could form a base from which other practical implementations and applications could be derived.

2. Mobile Applications:

There were mobile applications found that could detect drowsiness by monitoring a person's face. For example, the Drowsy Driver Android Application that can be found on Google Play Store. The major drawback of such applications is that they do not provide cross platform support. This limits the number of users that can have access to them. Additionally, they fail to communicate with the emergency contact of the driver and do not take into consideration the scenario of the driver needing support in case of an accident. Lastly, this application and similar ones had other hardware requirements in addition to just the camera, for example, 


\section{EPRA International Journal of Research and Development (IJRD)

higher camera resolution, audio output speakers etc.

\section{Uniqueness of this Approach.}

\section{1) Cross Platform:}

This project is completely based on accessing the camera of the device remotely. It is independent of the underlying platforms, such as Android, IOS etc. All of the functionalities which are provided are not platform specific and can be dispensed on any device irrespective of the operating system being used by that device.

\section{2) Device Independent:}

In addition to being cross platform, this project is designed in such a way that it does not matter what the monitoring device is, it can be a cell phone, a tablet etc. As long as the device supports the minimum requirements of having a camera, calling features and a stable internet connection, this project can be used to successfully alert a sleepy driver and prevent accidents.

\section{3) Simplicity:}

One of the major aspects of this project is that it is kept as simple as possible in terms of its requirements and implementation. All it needs is a device with an integrated camera, active internet connection and calling features, which most of the devices these days already possess. Additionally, the algorithms are designed to be implemented using only the libraries and programming concepts that are absolutely required and relevant to alert the driver. Not only does this project successfully avoid the unwanted complexities, but also makes it simple and convenient for the user to understand and use.

\section{4) Remote Monitoring:}

This project aims at providing remote monitoring of the driver. So, there can be someone else sitting at a different location that can monitor the driver while the driver is driving the vehicle. Since most of the drowsy driver accidents happen among youngsters, the remote interface can be used by parents to keep an eye on their kid while he/she is driving. Further, the remote access to the interface can be handed over to someone else before planning a late-night drive as in the case of commercial truck and bus drivers.

\section{5) Victim Support:}

By messaging the emergency contact of the driver, this project makes him/her check up on the driver by letting him/her know that the driver is not in a stable state to be driving the vehicle. In case if the driver gets into an accident, quick assistance can be provided by the emergency contact and the driver can be taken care of. Hence, this application takes into consideration the aspect of providing assistance to the victim if he/she ends up getting into an accident due to drowsy driving.

\section{AIMS AND OBJECTIVES}

A. Reduce the number of vehicle accidents that happen all around the world because of drowsy driving by keeping the driver alert and awake at all times.

B. Assist the commercial drivers such as truck drivers or bus drivers who drive long distances overnight for hours.

C. Examine the face of the driver at all times while he/she is driving.

D. Monitor the driver's eyes and measure the extent to which they are open or close.

E. To place a call on the driver's registered mobile number when the driver is found to be falling asleep.

F. To send a text message to the driver's emergency contact when the driver is found to be sleepy, informing them to contact the driver.

G. To render real-time accurate eye-aspectratio results based on the captured frames from the video.

H. To make the project easy to understand and use for the users.

I. To make the algorithm capable of differentiating sleepiness from just regular eye blinking.

J. To make the algorithm as computationally cheap as possible for the devices that support them.

\section{MECHANISM}

\section{A. Overview.}

The project implementation can be well understood by the flowchart given below. 


\section{EPRA International Journal of Research and Development (IJRD)

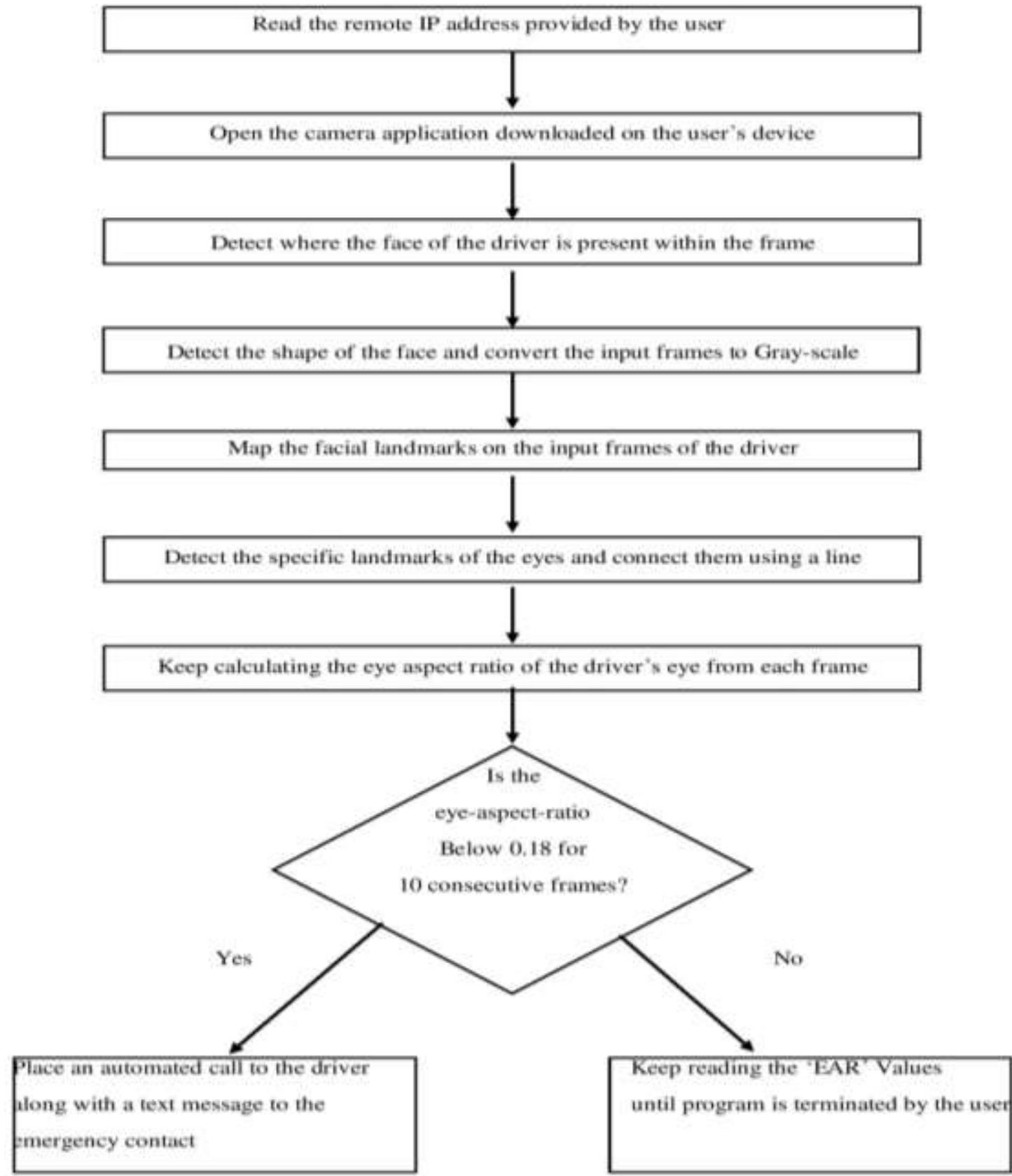

Chart 1. Step-wise Implementation of the Project.

As seen above, the input video stream of the driver is captured through the camera present on the device. The algorithm detects the face of the driver present in the video stream. Then different landmarks are plotted on the detected face of the driver to predict the shape of different parts of the face such as eyes, nose, lips, chin and jawline. Algorithm then computes the eye-aspect-ratio by using the landmarks plotted on the eyes.

\section{B. Technical Details.}

Calculating Eye Aspect Ratio:

To calculate the Eye Aspect Ratio, it is important to isolate the eye portion of the face. This is made possible by importing the facial landmark detection file in our code. The file consists of predefined points for extracting essential facial features that can be worked upon by the python code. The figure shown below gives a visual representation of the predefined points in the file. 


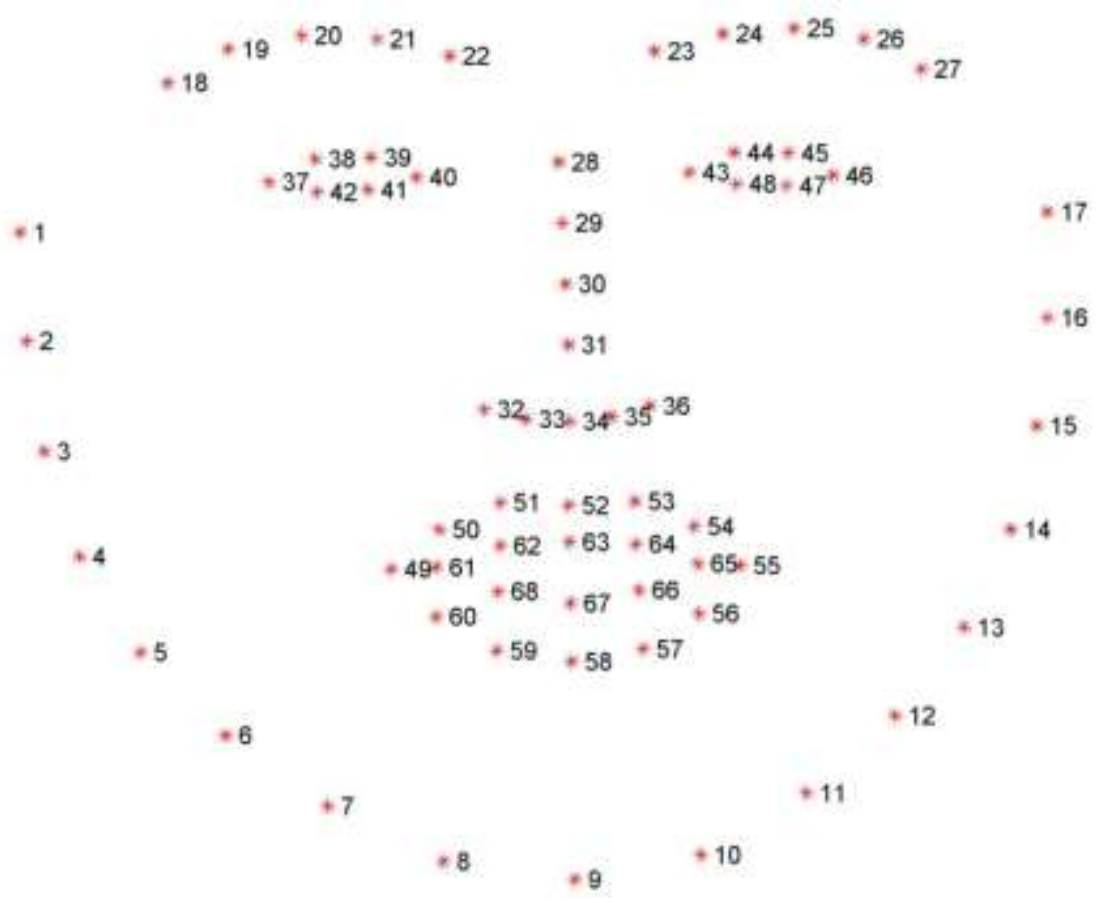

Figure 1. Visualizing the 68 facial landmark coordinates from the iBUG 300-W dataset.

Our project mainly focuses on the eye portion of the implementation. To calculate the Eye Aspect Ratio, driver. Thus, we use points $37-48$ for our consider figure 2 given below:

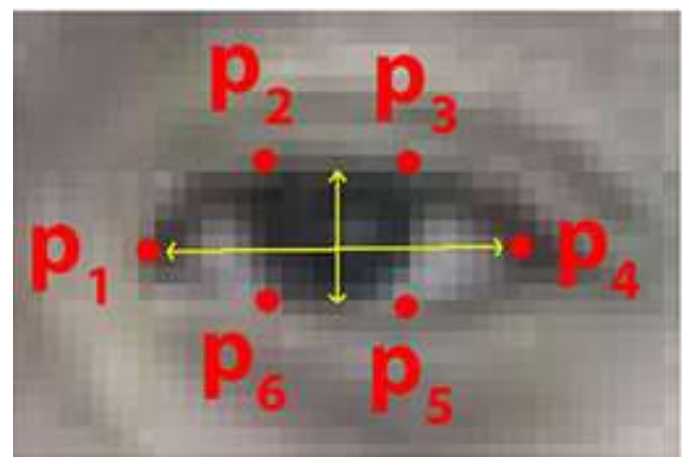

Figure 2. A visualization of eye landmarks when the eye is open.

- We first calculate the Euclidean distance between the points $\mathrm{P} 2$ and $\mathrm{P} 6$. Let $\mathrm{x}$ hold the value of Euclidean distance between points P2 and P6.

- Then we calculate the Euclidean distance between the points P3 and P5. Let y hold the value of Euclidean distance between points P3 and P5.

- Let $\mathrm{z}$ be the average of $\mathrm{x}$ and $\mathrm{y}$, i.e., $\mathrm{z}=(\mathrm{x}+$ y) $/ 2$.
- Now we calculate the Euclidean distance between the points P1 and P4. Let $\mathrm{w}$ hold the value of Euclidean distance between points $\mathrm{P} 1$ and $\mathrm{P} 4$.

- Finally, the Eye Aspect Ratio $=\mathrm{z} / \mathrm{w}$.

It is noticed that as the driver tends to be sleepy and his eyes close, the eye-aspect-ratio tends towards 0 . As soon as the Eye Aspect Ratio falls below 0.18 for 10 consecutive frames, our algorithm alerts the driver and sends a text message to the emergency contact. Refer to figure 3 and chart 2 given below. 


\section{EPRA International Journal of Research and Development (IJRD)}

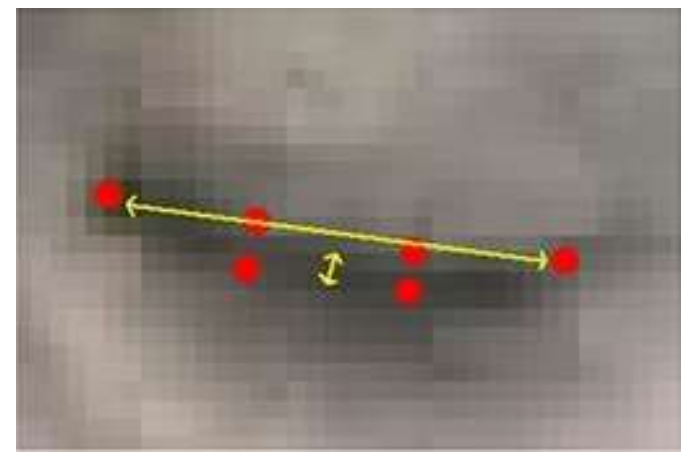

Figure 3. A visualization of eye landmarks when the eye is closed

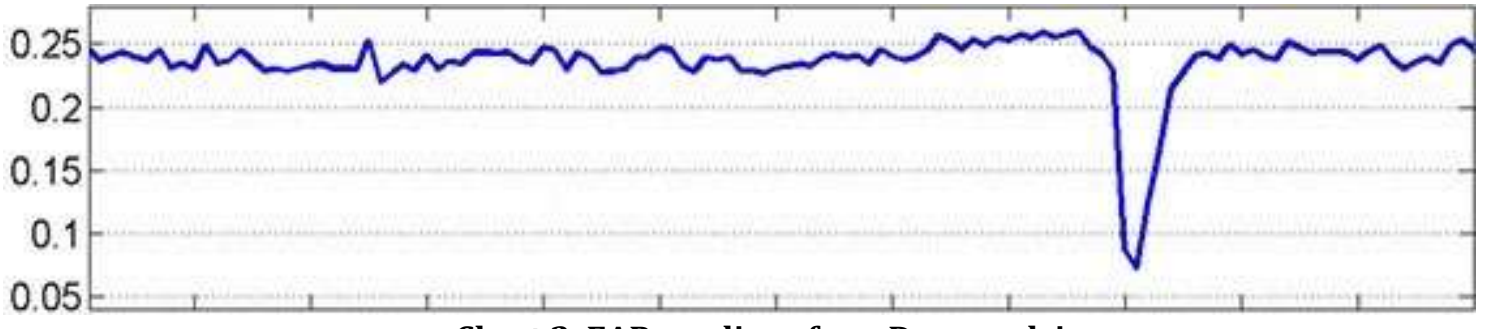

Chart 2. EAR readings for a Drowsy driver

C. Tasks that are needed to be done before driving.

The driver needs to make sure that the python script is executed on the desktop/laptop where the code has been installed and should keep the code running throughout the travel period of the driver. The main component for implementing the project is the device camera that can be accessed from remote locations. For this purpose, the driver needs to download any camera application that provides a specific IP address for accessing the device camera. This unique IP address needs to be updated in the code as no two users can have the same IP address for a camera. The driver should make sure that the device has a stable internet connection and is not in silent mode. He/she needs to turn on the camera application and should place the device (on which the application is running) in such a way that the face of the driver is seen properly.

\section{REQUIREMENTS}

A. Hardware Requirements.

1) Integrated Web Camera: A camera having configuration of more than 2 MP would work. The camera should be kept working throughout the travel period of the driver in a way that the driver's face can easily be recognized and monitored.

2) Integrated Device Speakers: It is important for the driver to ensure that the device is not in silent mode, and that the inbuilt speakers of the device works properly. Speakers are used to alert the driver as and when he/she would fall asleep.

3) RAM: The computer/laptop should have at least 2 GB RAM whereas the device monitoring the face of the driver should have at least 1 GB RAM. A higher RAM configuration is always preferred as it reduces the response time of the working system which would lead to a faster and a better performance of the overall system.

B. Software Requirements.

1) Operating System: Any operating system that supports Python can be used. However, it is advisable to use Ubuntu because of the ease with which it works with python libraries like Dlib, Cmake and OpenCV.

2) Programming Language: The project is built completely on Python. Any version of Python after Python 3.8 can be used. Some of the important libraries that we have incorporated and utilized in our project are:

- Dlib: Dlib is a modern $\mathrm{C}++$ toolkit containing machine learning algorithms and tools for creating complex software in $\mathrm{C}++$ to solve real world problems.

- OpenCV (Open-Source Computer Vision Library): It is a library of programming functions mainly aimed at real-time computer vision.

- CMake: It acts as a dependency for Dlib library. CMake is an open-source, crossplatform family of tools designed to build, test and package software. 


\section{EPRA International Journal of Research and Development (IJRD)

- NumPy: It is a library that is used to compute complex mathematical operations on arrays.

- SciPy: SciPy is a free and open-source Python library used for scientific computing and technical computing.

- Twilio: It is a library that provides calling and messaging facilities to the Python programming language.

3) Text Editor: Any text editor that supports Python programming language can be used.

\section{CONCLUSION}

So, in this way we provided an IOT based approach for monitoring the face of the driver while he or she is driving the vehicle. This project is successfully able to run machine learning algorithms to predict if the driver is about to fall asleep or not and render real time accurate results. This project was able to further use these results to alarm the driver and prevent him/her from falling asleep by generating an instant automated voice call to the driver's registered cell phone number. Additionally, an instant text message is sent to the driver's emergency contact so as to assure that there is someone to check up on the driver in case the driver happens to run into an accident.

This project is a unique implementation towards detecting drowsy driving as it offers an approach to remotely monitor the driver. It makes the implementation not only cross platform, but also device independent. As long as any device has an integrated camera, active internet connection and calling features, it can be used to implement this approach. This allows maximum users to benefit from it since the user need not worry about the kind of devices they have and the operating software supported by the device. This approach towards drowsiness detection has a lot of scope, especially for providing assistance to the different general and commercial drivers that are required to drive long distances overnight. Also considering the statistics indicating how many accidents are caused due to drowsy driving and lack of a current good implementation to prevent accidents due drowsydriving, this is a needed requirement for any driver who has a chance of falling asleep while driving.

\section{A. Future Scope.}

We intend to improvise on our project by adding more functionalities to it like a GPS feature which could send the live location of the driver to the emergency contact so that if an accident occurs, the driver can be located without any hassle and can be provided with medical treatment at the earliest.

Further, this project can be tied up with different travel and transport companies that carry people to their destination. Be it small local travel companies or even big ones like Uber or Ola can be collaborated with this project. This could be a great security feature for whoever is wanting to implement it. Additionally, the driver could be directed to nearby locations where the driver could get some rest. Also, algorithms which would detect drowsiness by considering the number of yawns of the driver while driving could be integrated with this project.

The overall motive of designing this project is to save maximum lives possible by avoiding accidents that are caused due to drivers falling asleep.

\section{REFERENCES}

1. dlib $C++$ Library. (n.d.). http://dlib.net/.

2. Drivers are Falling Asleep Behind the Wheel. National Safety Council. (n.d.). https://www.nsc.org/road-safety/safetytopics/fatigued-driving.

3. From our blog. CMake. (n.d.). https://cmake.org/.

4. Hoyoung, L., Rosebrock, A., Jalil, A., Harsha, Shukla, A., Paruchurisaikrishna, N.Trewartha, Vikram, Smith, Alexon, Kenny, Hitesh, Fang, Balesh, Ss, Roy, P., Cao, G., Marco, Carlos, ... (Faster) Facial landmark detector with dlib PyImageSearch says: (2021, April 17). Drowsiness detection with OpenCV. PyImageSearch. https://www.pyimagesearch.com/2017/05/08/drowsi ness-detection-opencv/.

5. Insurance Information Institute. (n.d.). Facts + Statistics: Drowsy driving. III. https://www.iii.org/fact-statistic/facts-statisticsdrowsy driving.

6. Kumari, K. Review on Drowsy Driving: Becoming Dangerous Problem, International Journal of Science and Research (IJSR), Jan. 2014, www.ijsr.net/archive/v3i1/MDIwMTM3MjI=.pdf.

7. McClafferty, J., Perez, M., Fang, Y., Guo, F., \& Dingus, T. A. (2018, June 13). Prevalence of Drowsy Driving Crashes: Estimates from a LargeScale Naturalistic Driving Study. AAA Foundation. https://aaafoundation.org/prevalence-drowsydriving-crashes-estimates-large-scale-naturalisticdriving-study/.

8. National Centre for Statistics and Analysis. (2020, December). Overview of motor vehicle crashes in 2019. (Traffic Safety Facts Research Note. Report No. DOT HS 813 060). National Highway Traffic Safety Administration

9. Safety, A. A. A. F. for T. (2020, June 23). 2019 Traffic Safety Culture Index. AAA Foundation. https://aaafoundation.org/2019-traffic-safetyculture-index/.

10. Wikipedia Foundation. (2021, April 13). SciPy. Wikipedia. https://en.wikipedia.org/wiki/SciPy.

11. Wikipedia Foundation. (2021, March 14). OpenCV. Wikipedia. https://en.wikipedia.org/wiki/OpenCV.

12. Wikipedia Foundation. (2021, May 26). NumPy. Wikipedia. https://en.wikipedia.org/wiki/NumPy. 